\title{
Kritische Orientierungen in der Geographie: Anmerkungen zur Studienmotivation und Berufswahl
}

\author{
Simon Runkel ${ }^{1}$ \\ Eingegangen: 23. April 2020 / Überarbeitet: 17. September 2020 / Angenommen: 19. September 2020 / Online publiziert: 8 . Oktober 2020 \\ (c) Der/die Autor(en) 2020
}

\section{Zusammenfassung}

Im vorliegenden Beitrag wird ausgehend von der Frage nach der Studienmotivation in der Geographie erörtert, inwieweit das Hochschulfach Geographie ein Orientierungsfach darstellt und damit auch Möglichkeit zur Kritik in gesellschaftlichen Zusammenhängen gibt. Geographie zu studieren, bedeutet im beruflichen und persönlichen Lebensvollzug eine Antwort darauf zu finden, was die Geographie ist. Im Studium wird eine berufliche Sinngebung ermöglicht und erprobt, weswegen im vorliegenden Beitrag argumentiert wird, dass diesen Sinngebungsprozessen eine dezidiert kritische Orientierung zuträglich ist. Mit Rückgriff auf Anne Buttimer wird aufgezeigt, in welchen Haltungen berufliche Sinngebungen einerseits bereits im Studium der Geographie eingeübt bzw. durch die Hochschullehre befördert werden können und wie dies disziplingeschichtlich mit Denkstilen im Fach zusammenhängt. Ferner wird erläutert, was Orientierung bedeutet und wie dies mit Kritik zusammenhängt. Es wird abschließend mit dem Vorschlag, Kritik als Mediation zu verstehen, für die Notwendigkeit einer kritisch angewandten Geographie plädiert.

Schlüsselwörter Sozialgeographie $\cdot$ Kritische Geographie $\cdot$ Geographiestudium · Planung · Kritik · Disziplingeschichte

\begin{abstract}
Proceeding from the question of what motivates someone to select geography as their field of study, the article debates whether geography as a university subject can be considered orientational and how this opens up the opportunity for social criticism. By studying geography, answers on what geography means are found through professional and personal conduct. During the course of studies, vocational meaning is made possible and is tested. The current article argues that a decidedly critical orientation is beneficial to these meaning processes. With reference to Buttimer (1984), the personal attitudes at play are illustrated by making sense of vocational meaning while studying geography and how this relates to styles of thought in the history of the discipline. Furthermore, the meaning of orientation can be explained and how this relates to criticism. By proposing to understand critique as mediation, the article concludes with a plea for critically applied geography.
\end{abstract}

\section{Warum ein Studium der Geographie?}

Untersuchungen zur Studienmotivation haben eine bedingte Aussagekraft. Einerseits bleibt unklar, warum sich Studieninteressierte gegen ein Geographie-Studium entscheiden. Andererseits ist erst ein systematischer Vergleich mit den Motivationen zur Wahl anderer Studienfächer aufschlussreich. Zudem lässt sich vermuten, dass es in biografischen

Simon Runkel

simon.runkel@uni-jena.de

1 Institut für Geographie, Friedrich-Schiller-Universität Jena, 07743 Jena, Deutschland
Auseinandersetzungen mit dieser Frage zu ,retrospektiven Fälschungen“" (Bartels und Hard 1975, S. 3) kommt. Insbesondere da die persönlichen Umstände stark differieren, die Umbrüche im Fach und seinem Image in Rechnung gestellt werden müssen und schließlich die reflektierte Auskunftsfähigkeit über die Studienmotivation unterschiedlich ausgeprägt ist. Dennoch deuten Untersuchungen an, dass neben einem spezifischen oder diffusen Interesse an der Geographie oder an beruflich vielversprechend erscheinenden Teilbereichen der Geographie, das Fach von Studieninteressierten als vielfältig, breit und abwechslungsreich wahrgenommen wird (Hard und Wenzel 1979; Klee und PiotrowskyFichtner 2003). 
Die Geographie gewinnt ihren Abwechslungsreichtum dadurch, dass sie ein interdisziplinäres Fach an der Nahtstelle zwischen Natur- und Sozialwissenschaft ist. Livingstone (1992, S. 177) hat dies das ,geographical experiment“ genannt, da „Natur“ und „Kultur“ unter einem Dach zusammengehalten werden. Die Spannung dieses Experiments führte stets neu zur Diskussion über die „Einheit der Geographie“ (Bartels und Hard 1975, S. 80; Schurr und Weichhart 2020). Neben der fachpolitischen Dimension dieser Debatte, zeigt sich die Spannung auch in der persönlichen Sinngebung, die im Laufe der geographischen Ausbildung gewonnen werden muss. Aufgrund der pluralen Identitäten der Disziplin - die Geographie als „Raum der Verbindungen" (Schurr und Weichhart 2020, S. 64) - verlangt die geographische Ausbildung den Studierenden eine persönliche Sinngebung ab (spätestens bei der Jobsuche). Selbst wenn aus einer Haltung der Unentschiedenheit oder, positiv gewendet, aus einer Haltung des vielseitigen Interesses, die Studienwahl getroffen wurde: Geographin und Geograph (sowie divers) zu werden, bedeutet, sich selbst im beruflichen und persönlichen Lebensvollzug eine Antwort darauf zu geben, was die Geographie ist. Die Notwendigkeit zur spezialisierten Entscheidung wird in das Studium der Geographie verlegt. Im Studium wird eine berufliche Sinngebung ermöglicht und erprobt, weswegen im vorliegenden Beitrag argumentiert wird, dass diesen Sinngebungsprozessen eine dezidiert kritische Orientierung zuträglich ist. Hierzu wird zunächst mit Rückgriff auf Anne Buttimer (1984) aufgezeigt, in welchen Haltungen berufliche Sinngebungen einerseits bereits im Studium der Geographie eingeübt werden können und wie dies disziplingeschichtlich mit Denkstilen im Fach zusammenhängt. Ferner wird dargestellt, wie die Geographie als ein Orientierungsfach verstanden werden kann. Abschließend wird für die Notwendigkeit einer kritisch angewandten Geographie plädiert.

\section{Berufliche Sinngebungen}

„Geographinnen und Geographen (sowie divers) können alles und nichts" ist eine Standardantwort auf die Frage, wofür ein Geographie-Studium gut ist. In Bezug auf berufliche Sinngebung eint Geographinnen und Geographen (sowie divers), dass die Antworten auf diese Frage eine groBe Bandbreite darstellen. Die Fähigkeit zum Umgang mit der Vielfalt ist eine zentrale Kompetenz der Absolventinnen und Absolventen (sowie divers). Hochschullehrerinnen und Hochschullehrer (sowie divers) sind jenseits der Vermittlung fachspezifischer Theorien, Inhalte und Methoden damit konfrontiert, Orientierung zu ermöglichen, was geographische Praxis für jede einzelne Person bedeuten kann.

Buttimer (1984, S. 34) unterscheidet auf Basis autobiografischer Zeugnisse von Geographinnen und Geogra- phen (sowie divers) in der beruflichen Sinngebung zwischen Poiesis, Paideia, Ergon und Logos. Diese hält sie als Auswahlmöglichkeit für Berufsrollen von Geographinnen und Geographen (sowie divers) für bedeutsam. Sie weist darauf hin, dass „eine einzelne Person mehr als nur eine Berufsrolle übernehmen kann und in der Regel auch übernimmt, was häufig von den Möglichkeiten und Zwängen ihrer Umgebung abhängt" (Buttimer 1984, S. 35). Berufliche Sinngebungen können biografisch in kreativer Weise verbunden werden, sie können sich überlagern und abwechseln. Sie werden vom sozialen Milieu, dem Hochschulangebot und von persönlichen Beziehungen mitgeprägt. Gleichwohl sie damit die bewusste rationale Optionalität von Berufsrollen gemäß ihrer individualistischen Handlungstheorie etwas überbetont und strukturelle Faktoren und Zwänge nicht beleuchtet, so bieten ihre Überlegungen eine interessante Heuristik zum Verstehen geographischer Berufsbiografien. $\mathrm{Zu}$ betonen ist, dass es mehr intersektionale Analysen benötigt, die sichtbar machen, dass Geographinnen und Geographen (sowie divers) keineswegs alle Türen offenstehen und auch die Disziplin selbst durch systematische Ausblendungen geformt wurde (Oswin 2020). Beruflicher Erfolg und Identitätsfindung verläuft für viele entlang spezifischer sozialer Privilegien, die mit dem Studium und den je eigenen Interessen wenig zu tun haben. Eine gendersensible, rassismuskritische und klassenbewusste Berufssoziologie des Fachs Geographie könnte Aufschluss geben, inwieweit der Studien- und Berufswahl rationale Entscheidungen oder kulturelle Stereotype zugrunde liegen (Finger et al. 2020, S. 2), die bei beruflichen Sinngebungsprozesse stets mitgedacht werden sollten.

Buttimer (1984) unterscheidet 4 Haltungen der Sinngebung in beruflichen Tätigkeiten. Die Haltung der Poiesis zeichnet sich als idiografische Suchbewegung aus, die (ortsbezogene) Ästhetik, Sinnlichkeit und Erfahrung betont. Sinn entsteht in der Kontemplation und Vertiefung der Welterfahrung. Für eine Angewandte Geographie, die sich auf das Lösen von Problemen richtet, ist eine solche Haltung scheinbar wenig tauglich. Doch insbesondere in der Planung lassen sich Berufsfelder identifizieren, in denen Absolventinnen und Absolventen (sowie divers) praxisrelevante Tätigkeiten in dieser kreativen Haltung übernehmen.

In der Haltung der Paideia gelingt Sinngebung durch die Vermittlung von Wissen, z.B. in Lehrberufen, oder in der Verbreitung von Wissen mittels anderer Tätigkeiten. Der hohe Anteil an Lehramtsstudierenden in der geographischen Hochschulausbildung zeugt von der Relevanz dieser beruflichen Sinngebung, die aber bedauerlicherweise in eklatantem Widerspruch zu den bildungspolitischen Einschränkungen steht, denen das Schulfach Erdkunde derzeit ausgesetzt ist (Kaube 2019).

In der Haltung des Ergon werden Probleme gelöst. Sinn entsteht in der Bearbeitung sozialer und ökologischer Her- 
ausforderungen. Die Sinngebung gelingt im Handeln. Aktuell erscheint dies angesichts der multiplen Krisen (z.B. Verschränkung von ökonomischen und ökologischen Krisen) von besonderer Wichtigkeit, z. B. wenn es um strategische Entscheidungen in der Regionalentwicklung geht.

In der Haltung des Logos ist die Vernunft vordergründig. Gefragt ist analytisches Denken zum Erkennen von Gesetzmäßigkeiten. Sinn entsteht in der rationalen Argumentation; wissenschaftliche Erkenntnisse erfordern dabei Objektivität. In der Haltung des Logos tritt z. B. derzeit in der Angewandten Geographie eine technokratische Weltsicht auf den Plan, die von einer gesellschaftspolitischen Neutralität digitaler Werkzeuge ausgeht.

In der Arbeit der Hochschullehrerinnen und Hochschullehrer (sowie divers) zeigt sich, dass sich die Geographie dadurch auszeichnet, dass sie nicht nur jene beruflichen Sinngebungen als buntes Nebeneinander zulässt, sondern dass sie in ihrem Selbstverständnis das polyvalente Miteinander als zentrales Ausbildungsziel hat. Ziel einer pluralistischen Geographie - die ihre Innovationskraft auch aus den disziplinären, gesellschaftlichen und geographischen „Randzonen“ (Schurr und Weichhart 2020, S. 64) gewinnt - ist es, dass Studierende lernen, zwischen verschiedenen Positionen zu vermitteln. Die Vielfalt der Disziplin führt zur Heterogenität der beruflichen Sinngebungsprozesse, die sich in der Lehre abbildet. Denn mit Vielfalt umzugehen, gilt nach wie vor als Erfolgsvoraussetzung von Geographinnen und Geographen (sowie divers) auf dem Arbeitsmarkt (Klemme et al. 2000).

Buttimer (1984, S. 35) deutet an, dass sich diese „Masken" in verschiedenen Paradigmen der Humangeographie unterschiedlich stark ausprägten. Hiervon zeugt auch die aktuelle Diskussion um die Identität der Disziplin (Schurr und Weichhart 2020). Dazu bedürfte es entsprechend auch einer mikrogeschichtlichen Auseinandersetzung mit den vielzähligen (Berufs-)Biografien von Geographinnen und Geographen (sowie divers) in den letzten Jahrzehnten. Für viele Geographinnen und Geographen (sowie divers) hat das Fach jenseits der Diskussionen zum Verhältnis von Geographie und Gesellschaft, Hochschule und Berufsfeld eine Schlüsselrolle in der persönlichen Sinngebung. Vor 50 Jahren stand die Geographie im Kontext einer gesellschaftspolitischen und wissenschaftlichen Entwicklung, die vom Glauben an Fortschritt, Wachstum, Planung und Steuerung geprägt war. Es formierte sich eine planungsaffine Humangeographie mit starkem Anwendungsbezug. Die berufliche Sinngebung war v.a. vom Logos und Ergon geprägt. Die Poiesis-Haltung der geographischen Landschaftsforschung wurde kritisiert. Geographinnen und Geographen (sowie divers) mit Diplom wurden als anwendende und staatstragende Sozialingenieurinnen und Sozialingenieure (sowie divers) benötigt. Die Raumplanung erlebte einen Boom: Flächennutzungs- und Bebauungspläne mussten erstellt werden und Interessen mussten abgewogen werden (v. Rohr 1994, S. 35).

Der Glaube an die Machbarkeit und Gestaltbarkeit der Zukunft geriet aber nicht zuletzt durch die sozialen, ökonomischen und politischen Krisen der 1970er-Jahre unter Druck. Berufliche Sinngebungen der Poiesis und Paideia traten erneut in den Vordergrund. Es entstand eine $\mathrm{Hu}-$ mangeographie, die sich mehr den Deutungsmustern und Lebenswirklichkeiten zuwendete (Pohl 1989). Damit konturierte sich eine, zunächst stark auf das Individuum ausgerichtete Geographie, die als Wegbereiterin einer Neuen Kulturgeographie gelten kann. Die Beschäftigung mit Theorien und Philosophien führte zu einer intellektuellen Pluralisierung, was aber auch zu Entfernungen und Entfremdungen innerhalb der Disziplin führte (Runkel und Everts 2017). Jüngst sind einerseits Ideen für eine erneuerte, stärker praxisorientierte Humangeographie (bzgl. Planung, Dünckmann et al. 2019) und andererseits auch für eine gesellschaftskritische Praxis entstanden. Eine solche Pluralisierung erfordert Orientierung.

\section{Geographie als Orientierungsfach}

Es liegt nahe, dass Geographie mit räumlicher Orientierung zu tun hat. Einerseits vollzog sich die Entwicklung der Geographie vom Fach zur wissenschaftlichen Disziplin entlang von angewandten Fragen der Orientierung und Navigation (Livingstone 1992, S. 32). Andererseits wurde (räumliche) Orientierung im Zuge der kognitivistischen Verengung der Wahrnehmungs- und Verhaltensgeographie psychologisiert (Gold 1980, S. 93). Phänomenologisch informierte Arbeiten nutzen aktuell den Begriff der Orientierung im Kontext politisch-geographischer Fragestellungen (Hannah 2019; Singh 2020). Schließlich ist jede geographische Exkursion eine Orientierungsübung (siehe Abb. 1). Im Folgenden widme ich mich der Frage, was es bedeuten kann, von der Geographie als Orientierungsfach zu sprechen. Obwohl hier keine systematische Begriffsbildung geschehen kann, wird eine kurze begriffliche Bestimmung der geographischen Metapher gegeben.

Mit Jaspers (1932, S. 85) könnte die Geographie wie andere wissenschaftliche Disziplinen zunächst als Wissenschaft der Weltorientierung gekennzeichnet werden. Allerdings zeigt sich in dieser anthropologischen Bestimmung, dass sich „Weltorientierung“ auf eine fundamentalontologische Gültigkeit beruft. Die Geographie als Erfüllungsgehilfin zur Orientierung der Erkenntnis an universalen Gültigkeiten wurde aber spätestens mit dem Aufkommen konstruktivistischer Perspektiven ad acta gelegt. Aus geographischen Tatsachen selbst, lässt sich keine Orientierung ableiten. Die Geographie bietet kein einheitliches Koordina- 


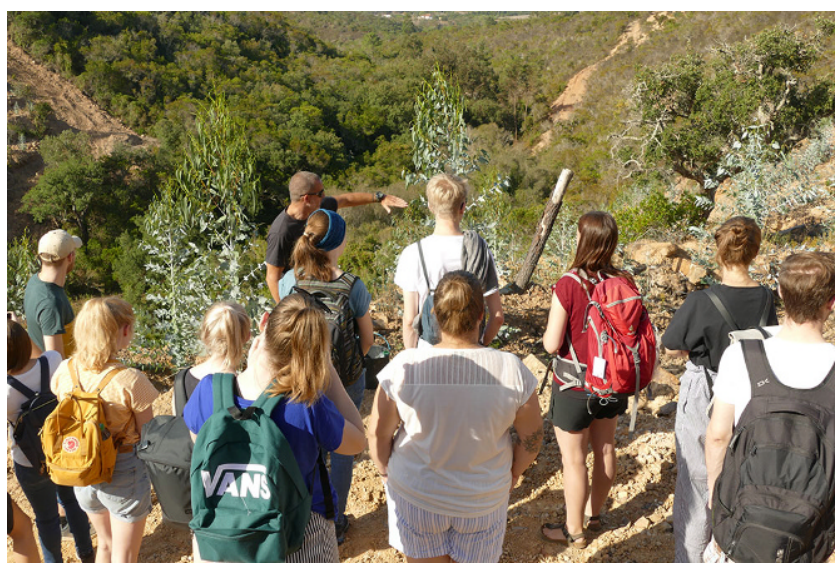

Abb. 1 Exkursionen mit Studierenden wie auf diesem Foto in Aljezur, Portugal schulen die Kompetenz, verschiedene Perspektiven einzunehmen. (Foto: Martin Franz)

tensystem, welches im Studienverlauf einfach zu entdecken wäre und zur Bestimmung der Sinngebung gereicht.

Stattdessen kann mit Stegmaier (2011) von einer „Autonomie der Orientierung “ gesprochen werden. Damit ist gemeint, dass „es die Orientierung selbst ist, die sich orientiert, daß das Selbst der Orientierung die Orientierung selbst oder kurz: daß eben die Orientierung als solche autonom ist" (Stegmaier 2011, S. 172). Die Orientierung erfüllt ein Bedürfnis der Vernunft und setzt sich ins Verhältnis zur umgebenden Welt (Stegmaier 2011, S. 173). Da jede Orientierung an eine vorhergegangene anschließt, so ist sie ursprünglich und richtet sich stets an sich selbst aus (Stegmaier 2011, S. 169-170). Stegmaier (2007, S. XVI): „Um einen Stadtplan benutzen zu können, muss man sich schon einigermaßen in Städten und mit Stadtplänen auskennen ... Orientierung setzt immer schon Orientierung voraus, Orientierung schließt immer schon an Orientierung an." Wichtig ist hierbei die Differenz zwischen Orientierung und Situation. Orientierung ist situativ. Neue bzw. krisenhafte Situationen erfordern die Sondierung von Handlungsoptionen. So ist Orientierung immer anders (Stegmaier 2011, S. 170). Orientierung muss in der Lage sein, sich auf unvorhersehbare Situationen und bewegliche Strukturen einzustellen (Stegmaier 2007, S. XVI). Ebenso wie der Begriff „Orientierung“ entstammt „Situation“ aus der Geographie: Mit „Situation“ (lat. „situs“) wird die Lage bzw. die Zuordnung von etwas zueinander bezeichnet. „Orientierung" bezeichnet die Ausrichtung. In der Erschließung der Situation wird ein Unterschied gemacht. Stegmaier (2007, S. 152) erläutert: „Die Korrelation von Situation und Orientierung ist die Unterscheidung, die zu Unterscheidungen führt." Zentral für Stegmaiers systemtheoretische Philosophie der Orientierung ist, dass er von Unterscheidungen ausgeht. Es gibt etwas nur unter der Bedingung, dass es als etwas unterschieden wird. Die Autonomie der Orientierung leitet er davon ab, dass Orientierung ,allem Beobachten nach Unterscheidungen vorausgeht, [und] alles Beobachten nach Unterscheidungen von vorausgehenden Orientierungsentscheidungen abhängt“" (Stegmaier 2011, S. 169). Stets werden in der autonomen Orientierung Spielräume für Entscheidungen eröffnet.

Zusammenfassend wird erstens deutlich, dass die Geographie selbst ein begriffliches Vokabular aufweist, das verstärkt konzeptionell in Wert gesetzt werden kann. Zweitens wird kenntlich, dass in der Geographie Orientierungsentscheidungen ermöglicht werden. Dies betrifft die beruflichen Sinngebungen, die im Studienverlauf ermöglicht werden. Etymologisch bedeutet „Sinn“ im Deutschen „Gang“, „Reise“ oder „Weg“ (Stegmaier 2007, S. 181). Sinn eröffnet sich immer erst in der Situation. „Sinn ist für die Orientierung das“, so Stegmaier (2007, S. 181), „womit sie ,etwas anfangen kann"“. Die Begriffsbestimmung zeigt, dass Orientierung der Anfang aller Entscheidungen ist (Stegmaier 2007, S. XV). Hierzu ist es notwendig, dass Orientierung sich in der Situation entscheidet. Die Situation stellt sich als Krisis (griech. „Entscheidung“, „Urteil“) dar. In der Krise ist Orientierung notwendig, denn damit es in der Situation zu einer Entscheidung kommen kann, muss zwischen Optionen vermittelt werden. Im Folgenden soll in diesem Sinne die Möglichkeit einer kritisch angewandten Geographie entfaltet werden.

\section{Warum eine kritische Orientierung notwendig ist}

Im Unterschied zu der Zeit als die Diplom-Studiengänge eingeführt wurden, kommen Absolventinnen und Absolventen (sowie divers) heute beruflich in Situationen, die davon gekennzeichnet sind, dass die Welt schon weitgehend fertig geplant ist (vgl. Blotevogel 2018). Von ihnen wird abverlangt, eine berufliche Sinngebung zu finden, die inmitten zunehmender politischer Polarisierung, entfesseltem Kapitalismus und drohender ökologischer Apokalypse nur wenig Spielraum zur Intervention zu bieten scheint. Die Anforderung an die Geographie als Orientierungsfach erscheint aktuell immens. Ein Ausweg aus der Ohnmacht gegenüber der Komplexität der spätmodernen Welt ist „Kritik als Praxis“" (Vobruba 2017). Dies zeigt sich in der Zunahme an Ansätzen, die sich als Kritische Geographie verstehen lassen. Eine angewandte kritische Geographie interveniert in Zusammenarbeit mit sozialen Bewegungen in gesellschaftliche Machtverhältnisse und politische Auseinandersetzungen hinein (Schipper 2017). Damit ermöglicht sich ein Transfer zwischen wissenschaftlicher Forschung und gesellschaftspolitischer Praxis.

Was ist mit Kritik gemeint? In den Sozialwissenschaften werden verschiedene Kritik-Begriffe diskutiert (Vobruba 2017). Ich möchte einen Kritik-Begriff vorschlagen, der 
es möglich macht, für berufliche Sinngebungen in der Geographie Orientierung zu leisten. Kritik muss nicht zwangsläufig als Opposition verstanden werden, obwohl es für ein solches Kritik-Verständnis gute Gründe gibt. Kritik kann auch als Mediation verstanden werden (Geiselhart 2020). Mediation heißt, vereinfacht gesagt, zwischen verschiedenen Positionen zu vermitteln. Kritik als Kunst der Beurteilung und der damit verbundenen Vermittlung zwischen konträren Entscheidungsmöglichkeiten ist im Sinne der Autonomie der Orientierung sehr wichtig. Kritik als Vermittlung bedeutet, Beziehungen zu analysieren, Interessen abzuwägen und diese an die jeweils andere Seite zu vermitteln. Kritik als Mediation erfordert die Kompetenz, sich auf neue Situationen produktiv einlassen zu können. Kritisch ist dieses Unternehmen insofern, dass es allen Seiten Grenzen aufzeigen muss und weil es auch sicherstellen muss, dass alle Seiten am Prozess der Mediation interessiert sind. Dafür braucht es ein Gespür für Machtasymmetrien in sozialen Beziehungen. Insbesondere für Fragen der angewandten Geographie ist aufgrund der zunehmenden sozialen Ungleichheiten eine Machtblindheit problematisch. Eine kritisch angewandte Geographie ist dadurch ausgezeichnet, dass sie sich in vermittelnder Weise den verschiedenen Positionen zuwendet, Widersprüche aufzeigt und an deren Auflösung arbeitet.

Dies ist nicht zwangsläufig ein Alleinstellungsmerkmal des Orientierungsfachs Geographie. Allerdings ist die Geographie besonders gut geeignet, kritische Orientierung zu bieten. Erstens aufgrund der Tatsache, dass sie vielseitig interessierten (oder unentschiedenen) Studierenden aufgrund ihres Abwechslungsreichtums eine umfassende Ausbildung bietet. Zweitens weil sie als Fach selbst, von Spannungen und Widersprüchen gekennzeichnet ist. Drittens weil durch die im Fach vermittelte Regionalkompetenz ein Gespür für sowohl globale als auch lokale Dynamiken vermittelt wird. Viertens weil die Heterogenität der beruflichen Sinngebungen in der Geographie, die Lehrveranstaltungen zu einer Arena divergierender Perspektiven macht, aufgrund dessen eine hohe Kompetenz der Mediation bereits im Studium geschult wird. Die geographische Ausbildung ist davon gekennzeichnet, dass verschiedene berufliche Sinngebungen im Studium aufeinandertreffen.

Im Sinne einer kritisch angewandten Geographie können Geographinnen und Geographen (sowie divers) in politische Entscheidungs- und Planungsprozesse als um die Notwendigkeit der globalen Veränderungen wissende Mediatorinnen und Mediatoren (sowie divers) hineinwirken. Angesichts der aktuellen Krisen benötigen wir eine solche Orientierung mehr denn je.

Danksagung Ich bedanke mich bei Klaus Kost zur Einladung auf die Sitzung der DVAG (Deutscher Verband für Angewandte Geographie) auf dem Kieler Geographie-Kongress 2019 sowie bei Wolf-Dietrich Sahr und Ferdinand Stenglein für hilfreiche Anmerkungen zu früheren
Versionen des Artikels. Mein ausdrücklicher Dank gilt auch den beiden anonymen Personen (m/w/d), die in den Gutachten inspirierende und konstruktive Kritik geübt haben.

Funding Open Access funding enabled and organized by Projekt DEAL.

Open Access Dieser Artikel wird unter der Creative Commons Namensnennung 4.0 International Lizenz veröffentlicht, welche die Nutzung, Vervielfältigung, Bearbeitung, Verbreitung und Wiedergabe in jeglichem Medium und Format erlaubt, sofern Sie den/die ursprünglichen Autor(en) und die Quelle ordnungsgemäß nennen, einen Link zur Creative Commons Lizenz beifügen und angeben, ob Änderungen vorgenommen wurden.

Die in diesem Artikel enthaltenen Bilder und sonstiges Drittmaterial unterliegen ebenfalls der genannten Creative Commons Lizenz, sofern sich aus der Abbildungslegende nichts anderes ergibt. Sofern das betreffende Material nicht unter der genannten Creative Commons Lizenz steht und die betreffende Handlung nicht nach gesetzlichen Vorschriften erlaubt ist, ist für die oben aufgeführten Weiterverwendungen des Materials die Einwilligung des jeweiligen Rechteinhabers einzuholen.

Weitere Details zur Lizenz entnehmen Sie bitte der Lizenzinformation auf http://creativecommons.org/licenses/by/4.0/deed.de.

\section{Literatur}

Bartels D, Hard G (1975) Lotsenbuch für das Studium der Geographie als Lehrfach. Selbstverlag, Bonn, Kiel

Blotevogel HH (2018) Geschichte der Raumordnung. In: ARL Akademie für Raumforschung und Landesplanung (Hrsg) Handwörterbuch der Stadt- und Raumentwicklung. ARL, Hannover, S 793-803

Buttimer A (1984) Ideal und Wirklichkeit in der Angewandten Geographie. Münchener Geographische Hefte, Bd. 51. Michael Lassleben, Kallmünz

Dünckmann F, Haubrich D, Runkel S (2019) Praktiken und Planung. In: Schäfer S, Everts J (Hrsg) Handbuch Praktiken und Raum. transcript, Bielefeld, S 317-339

Finger C, Solga H, Ehlert M et al (2020) Gender differences in the choice of field of study and the relevance of income information. Insights from a field experiment. Res Soc Stratif Mob 65:1-17

Geiselhart K (2020) Truth and academia in times of fake news, alternative facts and filter bubbles: a pragmatist notion of critique as mediation. In: Wills J, Lake R (Hrsg) The power of pragmatism: knowledge production and social inquiry. Manchester University Press, Manchester, S 139-156

Gold JR (1980) An introduction to behavioural geography. Oxford University Press, Oxford

Hannah M (2019) Direction and socio-spatial theory: a political economy of oriented practice. Routledge, London

Hard G, Wenzel HJ (1979) Wer denkt eigentlich schlecht von der Geographie? Geogr Rundsch 6:262-268

Jaspers K (1932) Philosophie. Philosophische Weltorientierung Bd. 1. Springer, Berlin

Kaube J (2019) Kampf ums Schulfach Erdkunde Frankfurter Allgemeine Zeitung. https://www.faz.net/aktuell/feuilleton/debatten/ geographie-der-kampf-um-das-schulfach-erdkunde-16538969. html. Zugegriffen: 10. Juli 2020

Klee A, Piotrowsky-Fichtner M (2003) Wer denkt heute schlecht von der Geographie? Standort Z Angew Geogr 4:179-183

Klemme M, Monheim H, Stegen R (2000) Geographie heute. Erfolgreiche Umorientierung auf die Praxis. Standort Z Angew Geogr 3:49-53

Livingstone DN (1992) The geographical tradition. Blackwell, Malden

Oswin N (2020) An other Geography. Dialog Hum Geogr 10(1):9-18 
Pohl J (1989) Die Wirklichkeiten von Planungsbetroffenen verstehen. Eine Studie zur Umweltbelastung im Münchener Norden. In: Sedlacek P (Hrsg) Programm und Praxis qualitativer Sozialgeographie. Wahrnehmungsgeographische Studien zur Regionalentwicklung, 6. BIS, Oldenburg, S 39-64

v Rohr GH-G (1994) Angewandte Geographie. Westermann, Braunschweig

Runkel S, Everts J (2017) Geographien sozialer Krisen / Krisen sozialer Geographien. Geogr Helv 72:475-482

Schipper S (2017) Wohnraum dem Markt entziehen? Wohnungspolitik und städtische soziale Bewegungen in Frankfurt und Tel Aviv. Springer VS, Wiesbaden
Schurr C, Weichhart P (2020) From Margin to Center? Theoretische Aufbrüche in der Geographie seit Kiel 1969. Geogr Helv 75:53-67

Singh AL (2020) Arendt in the refugee camp: The political agency of world-building. Polit Geogr 77:1-9

Stegmaier W (2007) Philosophie der Orientierung. De Gruyter, Berlin, New York

Stegmaier W (2011) Die Autonomie der Orientierung. In: Figal G (Hrsg) Internationales Jahrbuch für Hermeneutik, Bd. 10. Mohr Siebeck, Tübingen, S 161-173

Vobruba G (2017) Die Kritikkontroverse. Probleme der Unterscheidung von Praxis und Theorie. Soziologie 46(2):173-190 